\title{
DeepUbi: a deep learning framework for prediction of ubiquitination sites in proteins
}

\author{
Hongli Fu ${ }^{1 \dagger}$, Yingxi Yang ${ }^{1 \dagger}$, Xiaobo Wang ${ }^{1}$, Hui Wang ${ }^{2}$ and Yan $\mathrm{Xu}^{1,3^{*}}$ (1)
}

\begin{abstract}
Background: Protein ubiquitination occurs when the ubiquitin protein binds to a target protein residue of lysine $(K)$, and it is an important regulator of many cellular functions, such as signal transduction, cell division, and immune reactions, in eukaryotes. Experimental and clinical studies have shown that ubiquitination plays a key role in several human diseases, and recent advances in proteomic technology have spurred interest in identifying ubiquitination sites. However, most current computing tools for predicting target sites are based on small-scale data and shallow machine learning algorithms.

Results: As more experimentally validated ubiquitination sites emerge, we need to design a predictor that can identify lysine ubiquitination sites in large-scale proteome data. In this work, we propose a deep learning predictor, DeepUbi, based on convolutional neural networks. Four different features are adopted from the sequences and physicochemical properties. In a 10-fold cross validation, DeepUbi obtains an AUC (area under the Receiver Operating Characteristic curve) of 0.9 , and the accuracy, sensitivity and specificity exceeded $85 \%$. The more comprehensive indicator, MCC, reaches 0.78 . We also develop a software package that can be freely downloaded from https://github.com/Sunmile/DeepUbi.
\end{abstract}

Conclusion: Our results show that DeepUbi has excellent performance in predicting ubiquitination based on large data.

Keywords: Ubiquitination, Deep learning, Convolutional neural networks

\section{Background}

Ubiquitin was first discovered by Goldstein et al. in 1975 [1]. Ubiquitination, covalent attachment of ubiquitin to a variety of cellular proteins, is a common post-translational modification (PTM) in eukaryotic cells [2]. In the process of ubiquitination, ubiquitin is attached to substrates on lysine $(\mathrm{K})$ residues by a three-stage enzymatic reaction. There are three enzymes involved-ubiquitin activating enzyme (E1s), ubiquitin conjugating enzyme (E2s) and ubiquitin ligating enzyme (E3s), which work one after another [3-5]. The ubiquitination system is responsible

\footnotetext{
* Correspondence: xuyan@ustb.edu.cn

${ }^{\dagger}$ Hongli Fu and Yingxi Yang contributed equally to this work.

'Department of Information and Computing Science, University of Science and Technology Beijing, Beijing 100083, China

${ }^{3}$ Beijing Key Laboratory for Magneto-photoelectrical Composite and Interface Science, University of Science and Technology Beijing, Beijing 100083, China Full list of author information is available at the end of the article
}

for many aspects of cellular molecular function, such as protein localization, metabolism, regulation and degradation [4-7]. It also participates in the regulation of various biological processes such as cell division and apoptosis, signal transduction, gene transcription, DNA repair and replication, intracellular transport and virus budding $[4,5]$. Evidence has shown that ubiquitination has a close relationship with cell transformation, immune response and inflammatory response [8]. Abnormal ubiquitination status is also involved in many diseases. For example, the ubiquitination of metastasis suppressor 1 , mediated by the skp1-cullin1-F- box beta-transducin repeat-containing protein, is essential for regulating cell proliferation and migration in breast and prostate cancers [9].

Due to the roles of ubiquitination, the precise prediction of ubiquitination sites is particularly important. Conventional experimental methods are time-consuming and 
labour-intensive, and thus computational methods are necessary as a supplementary approach $[10,11]$. In recent years, a variety of machine learning methods have been applied to predict protein ubiquitination sites. Tung and Ho [12] developed a ubiquitination site predictor UbiPred, using support vector machine (SVM) with 31 informative physicochemical features selected from the published amino acid indices [13]. Radivojac [14] used a random forest algorithm to develop a predictor, UbPred, in which 586 sequence attributes were employed as the input feature vector. Zhao [15] adopted an ensemble approach to the voting mechanism. Lee [16] designed UbSite, which uses an efficient radial basis function (RBF) kernel to identify ubiquitination sites. Chen [17] proposed a predictor, CKSAAP_UbSite, using the composition of k-spaced amino acid pairs (CKSAAP). Cai [18] proposed a predictor utilizing the nearest neighbour algorithm. Chen [19] proposed a new tool, UbiProber, which was designed for general and specific species. Chen [20] developed hCKSAAP_UbSite by integrating four different types of predictive variables. Qiu [21] developed iubiq-lys using support vector machine. Cai and Jiang [22] used multiple machine learning algorithms to predict ubiquitination sites. Wang [23] designed a tool, ESA-UbiSite, using an evolutionary algorithm (ESA). In addition, there are many other predictors such as UbiSite [24], UbiBrowser [25], RUBI [26], the WPAAN classifier [27], MDDLogoclustered SVM models [28] and the non-canonical pathway network [29]. Although various ubiquitination site predictors have been developed, there are still limitations. As noted above, the existing computational methods for predicting ubiquitination sites are shallow machine learning methods and their datasets are small. However, a large amount of biomedical data has been accumulated and shallow machine learning algorithms do not handle big data well. In this study, we propose a lysine ubiquitination predictor, DeepUbi, using a deep learning framework on a large dataset.

\section{Results}

\section{Cross-validation performance}

For the series of hyperparameter choices, we obtain a set of better performing hyper-parameters, which are shown in Table 1. Using a set of clear and effective metrics defined in Eq. 4 to measure the quality of predictors, we considered how to objectively derive the values. Three different verification methods are generally used to evaluate the predictive performance: the independent dataset test, sub-sampling test and jackknife test [30]. The jackknife test can exclude the "memory" effect and the arbitrariness problem because the outcome obtained by the jackknife cross-validation is always unique for a given benchmark dataset [21]. However, it is time-consuming, especially for big datasets. In this study, $\mathrm{k}$-fold cross
Table 1 The values of super-parameter tuning

\begin{tabular}{ll}
\hline Super-Parameter & Preferred Setting \\
\hline Embedding length & 21 \\
Batch size & 65 \\
Maximum epoch & 30 \\
Convolution blocks & $([2-6], 64$, ReLU) \\
Fully connected layer units & 128 \\
Cutoff & 0.5 \\
Dropout & 0.7 \\
Learning rate & 0.01 with decay rate 0.95 \\
Regularization & L2 \\
\hline
\end{tabular}

validation was utilized to evaluate the performance of the proposed predictors because of the large dataset.

First, the 4-fold, 6-fold, 8-fold and 10-fold cross validations are executed 10 times on the simple One-Hot encoding scheme. The results are shown in Table 2. All of the accuracies are greater than $85 \%$ and the highest accuracy reaches $88.74 \%$, illustrating the robustness of the CNNUbi. The ROC curves and AUC values are shown in Fig. 1 and are more intuitive, and the largest AUC value was 0.89 . These results show that the deep learning framework learns some instinct information and has good performance. To obtain more information, we add three other features into the One-Hot encoding scheme (see Table 3 and Fig. 2). In the 10-fold cross-validation, all the ROC curves are very close to each other. The One-Hot plus CKSAAP encoding scheme clearly performs the best in all of these features. We call it DeepUbi with an AUC of 0.9066 and MCC of 0.78 .

Our DeepUbi predictor was obtained using balanced data. In the experimentally verified ubiquitination and non-ubiquitination data, the ratio of positive and negative peptides was 1:8. We also tested the performance on naturally distributed data when the algorithm was trained with balanced data. The results in Table 4 illustrate that the performance is slightly worse than with balanced data.

\section{Comparison with other existing methods}

A comprehensive comparison of our models with the available sequence-based predictors was performed and the corresponding data and results are shown in Table 5 .

Table 2 The results of 4-, 6-,8-, 10 -fold cross-validations with the One-Hot feature

\begin{tabular}{llllll}
\hline Cross-Validation & Acc (\%) & Sn (\%) & Sp (\%) & AUC & MCC \\
\hline 4-fold & 86.86 & 84.33 & 89.57 & 0.8787 & 0.74 \\
6-fold & 88.47 & 86.65 & 90.43 & 0.8853 & 0.77 \\
8-fold & 88.06 & 88.26 & 87.84 & 0.8884 & 0.76 \\
10-fold & 89.58 & 87.65 & 91.65 & 0.8905 & 0.79 \\
\hline
\end{tabular}




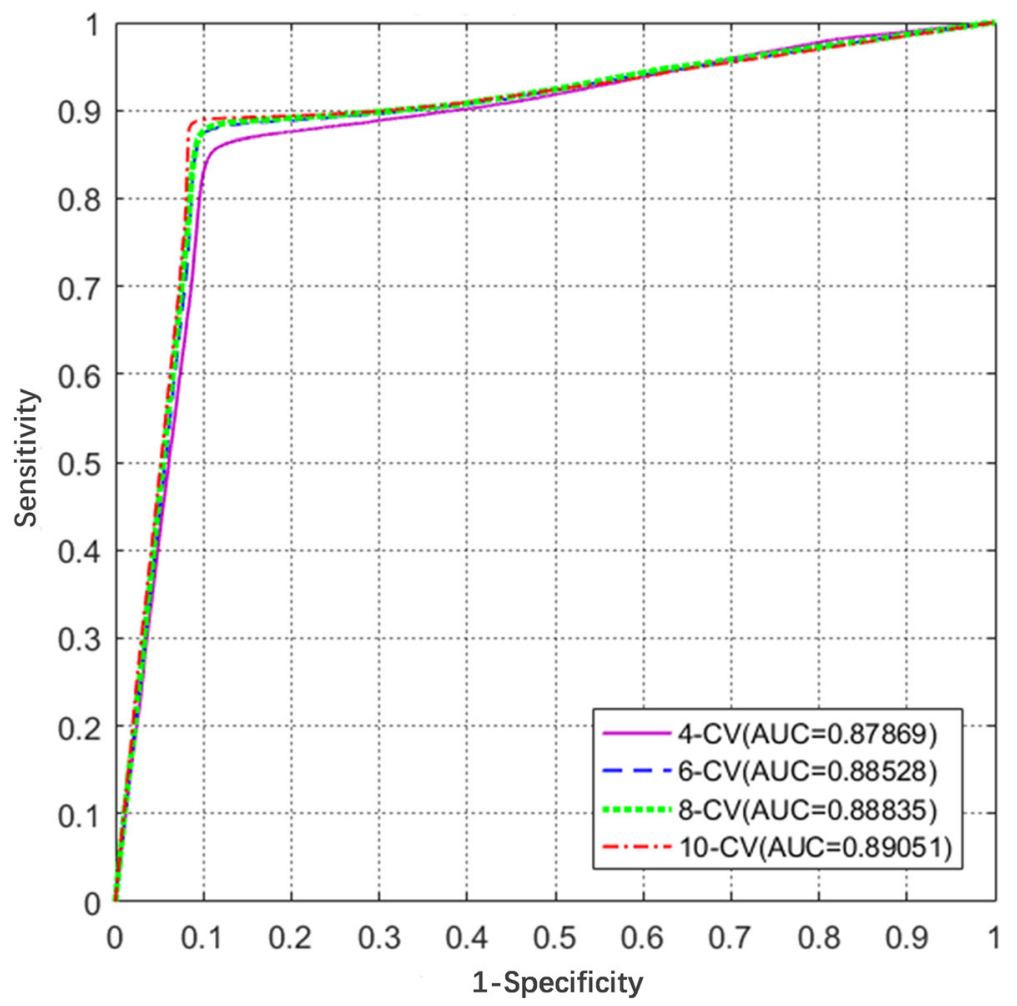

Fig. 1 ROC curves of different cross-validations. ROC curves and their AUC values of 4-, 6-, 8-, and 10-fold cross validations with the One-Hot encoding scheme

In the last decade, many researchers have contributed to the prediction and research of ubiquitination sites in proteins. The comparison shows that the deep learning model performs very well on big datasets. The predictors improved the accuracy by adding new features, using a variety of machine learning algorithms or adding new datasets. The precision of the predictors is approximately 0.8 . In this study, we propose the DeepUbi predictor and apply a deep learning framework with more accuracy. The AUC close to 0.9 and other indicators of accuracy, sensitivity and specificity are also better than those of existing methods. These results suggest that DeepUbi learned deeper characteristics.

To eliminate the impact of data volume differences and make a more vivid comparison, we conduct additional experiments. We randomly select the same number of positive and negative samples as the existing

Table 3 The results of four different encoding schemes in the 10-fold cross-validation

\begin{tabular}{llllll}
\hline Features & Acc (\%) & Sn (\%) & Sp (\%) & AUC & MCC \\
\hline One-Hot & 89.58 & 87.65 & 91.65 & 0.8905 & 0.79 \\
One-Hot + CKSAAP & 88.98 & 89.80 & 88.10 & 0.9066 & 0.78 \\
One-Hot + PseAAC & 86.05 & 87.58 & 84.41 & 0.8847 & 0.72 \\
One-Hot + IPCP & 86.41 & 83.44 & 89.61 & 0.8932 & 0.73 \\
\hline
\end{tabular}

predictor from our data 10 times. Each sample set is tested with 10 cross-validations, and the average results are listed in Table 6. Comparison of Table 5 and Table 6 shows that the DeepUbi results are much higher than those of other predictors for the same number of samples. For example, the data in UbiPred has an Acc of $84.44 \%$, Sn of $83.44 \%$, Sp of $85.43 \%$, AUC of 0.85 and MCC of 0.69. Selecting the same number UbiPred data as the test set 10 times, the average result for DeepUbi is an Acc of $98.77 \%$, Sn of $98.87 \%$, Sp of $98.67 \%$, AUC of 0.99 and MCC of 0.98. The AUC values of DeepUbi are close to 0.9, illustrating the performance of deep learning.

\section{Analysis of ubiquitination peptides}

To illustrate the performance of our predictor, we also conduct an analysis using the training data. First, the probabilistic histogram of composition of flanking amino acids surrounding the ubiquitination candidate sites is generated, as shown in Fig. 3a and b. Amino acid residues Ala (A), Glu (E), Leu (L), Arg (R) and Ser (S) appear more ratio in positive data (ubiquitination fragments), while Cys $(\mathrm{C})$, Phe $(\mathrm{F})$, His $(\mathrm{H})$, Ile $(\mathrm{I})$ and Val $(\mathrm{Y})$ are more enriched in negative data (non-ubiquitination fragments). Next, a well-known tool, Two Sample Logo [31], is applied to detect the position-specific amino acid composition 


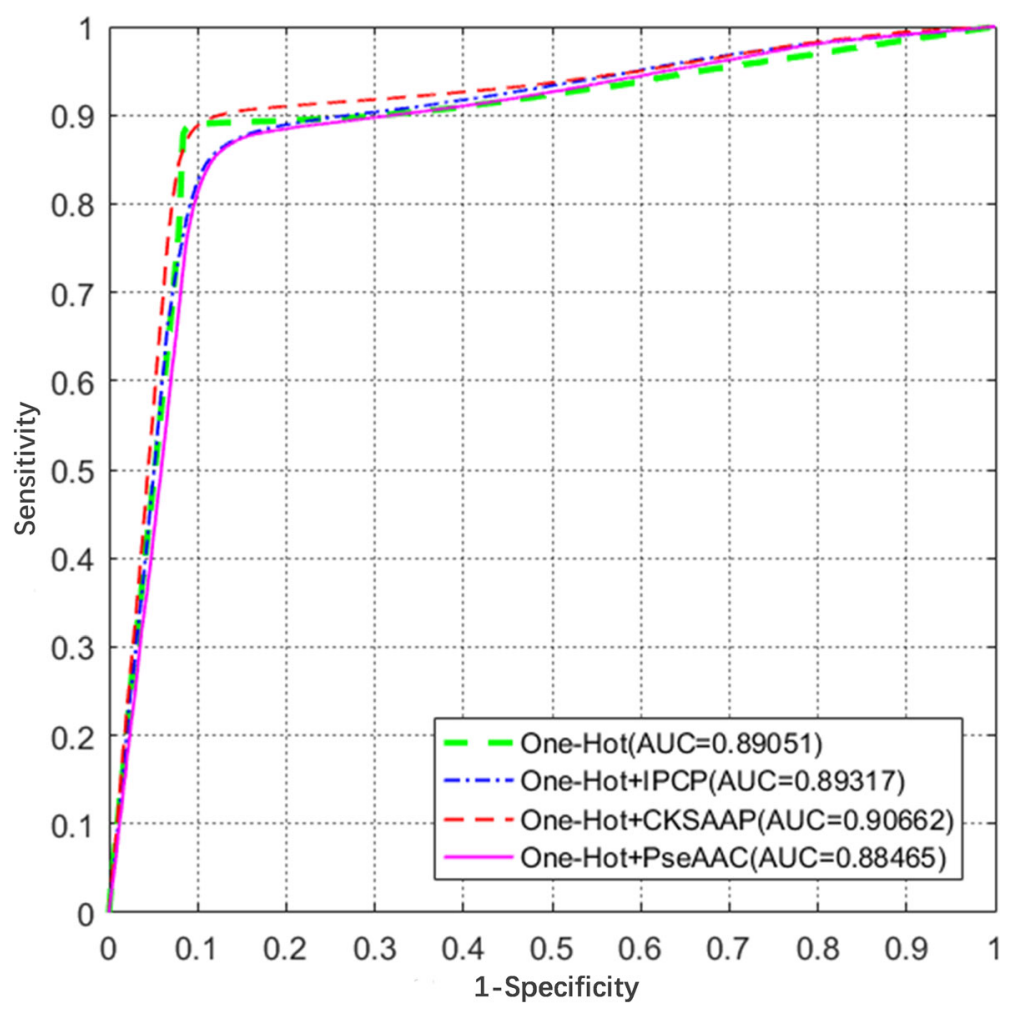

Fig. 2 ROC curves of different feature constructions. ROC curves and their AUC values of four features in the 10-fold cross validation. These curves are very close to each other which illustrate the robustness of the model

difference between the training data, and the sequence logo is shown in Fig. 3c. The results reveal the dependencies of flanking amino acids around the substrate sites.

\section{Discussion}

We use the biggest data repository designed for protein lysine modification to learn the DeepUbi predictor. A convolutional neural network, a deep learning framework, is adopted to predict ubiquitination. It is composed of a convolutional layer, a nonlinear layer and a pooling layer. Convolutional neural networks can learn a large number of mapping relations between input and output without any precise mathematical expression between the input and output. We construct six steps, including inputting the fragment, constructing an embedding layer, building multi-convolution-pooling layers, adding features, constructing fully connected layers, and the output layer. The deep learning framework is first used to predict ubiquitination.

Table 4 The results for naturally distributed DeepUbi data

\begin{tabular}{lllllll}
\hline No. of fragments & Acc (\%) & Sn (\%) & Sp (\%) & AUC & MCC & Pos:Neg \\
\hline 900 & 50.56 & 45.50 & 91.00 & 0.5490 & 0.23 & $1: 8$ \\
9000 & 49.56 & 44.46 & 90.30 & 0.6626 & 0.22 & $1: 8$ \\
\hline
\end{tabular}

Four better encoding schemes are adopted in the feature construction, One-Hot encoding, the physicochemical properties, the composition of $\mathrm{k}$-spaced amino acid pairs (CKSAAP) and the pseudo amino acid composition. One-Hot plus CKSAAP have the best performance with and AUC of 0.9066 in the cross-validation.

In the data, the sequence motif analysis shows that there are differences between positive and negative fragments. Thus, it is feasible to obtain classification information from the peptide itself. Different features are adopted to train the model. The hybrid of One-Hot and CKSAAP is selected as the best, with an AUC of 0.9066.

DeepUbi has better performance than the existing tools. Researchers could use the predictor to select potential candidates and conduct experiments to verify them. This will reduce the range of candidate proteins and save time and labour. The sequence analysis of the ubiquitination will provide suggestions for future work.

In the future, we will investigate other feature constructions that may better extract the properties of samples. Second, we aim to improve performance by increasing the depth and model parameters through system learning. The current method may also be used to identify other PTM sites in proteins. 
Table 5 Comparison of DeepUbi and other ubiquitination prediction tools

\begin{tabular}{|c|c|c|c|c|c|c|}
\hline Predictor & No. of positive samples & Acc (\%) & $\operatorname{Sn}(\%)$ & $S p(\%)$ & $\mathrm{AUC}$ & MCC \\
\hline UbiPred & 151 & 84.44 & 83.44 & 85.43 & 0.85 & 0.69 \\
\hline UbPred & 265 & 72.0 & - & - & 0.79 & - \\
\hline UbSite & 385 & 74.5 & 65.5 & 74.8 & - & - \\
\hline CKSAAP_UbSite & 263 & 73.4 & 69.85 & 76.96 & 0.81 & 0.47 \\
\hline UbiProber & 22,192 & - & 37.0 & 90.0 & 0.77 & 0.63 \\
\hline hCKSAAP_UbSite & 9537 & - & - & - & 0.77 & - \\
\hline iUbiq-Lys & 659 & 82.14 & 80.56 & 99.39 & - & 0.50 \\
\hline ESA-UbiSite & 85 & 94.0 & 96.0 & 92.0 & - & 0.92 \\
\hline DeepUbi & 53,999 & 88.98 & 89.80 & 88.10 & 0.91 & 0.78 \\
\hline
\end{tabular}

\section{Conclusion}

In this work, we propose a new ubiquitination predictor, DeepUbi, which uses a deep learning framework and achieves satisfactory success with the biggest data set. DeepUbi extracts features from the original protein fragments with an AUC of 0.9066 and an MCC of 0.78. We construct six steps including inputting fragment, constructing an embedding layer, building multi-convolution-pooling layers, adding features, constructing fully connected layers, and output layer. The deep learning framework is first used in prediction of ubiquitination. However, DeepUbi is not too deep, as we only use two convolution-pooling structures. We also develop a software package for DeepUbi that can be freely downloaded from https://github.com/Sunmile/ DeepUbi. The deep learning model is an effective prediction method and will improve accuracy by increasing the depth in the future.

Table 6 The DeepUbi results for the same number of samples as the other existing tools

\begin{tabular}{llllll}
\hline No. of positive samples & Acc (\%) & Sn (\%) & Sp (\%) & AUC & MCC \\
\hline UbiPred & 84.44 & 83.44 & 85.43 & 0.85 & 0.69 \\
DeepUbi & 98.77 & 98.87 & 98.67 & 0.9993 & 0.98 \\
UbPred & 72.0 & - & - & 0.79 & - \\
DeepUbi & 98.51 & 98.45 & 98.57 & 0.9975 & 0.97 \\
UbSite & 74.5 & 65.5 & 74.8 & - & - \\
DeepUbi & 97.99 & 97.79 & 98.18 & 0.9933 & 0.96 \\
CKSAAP_UbSite & 73.4 & 69.85 & 76.96 & 0.81 & 0.47 \\
DeepUbi & 99.19 & 98.96 & 99.42 & 0.9959 & 0.98 \\
UbiProber & - & 37.0 & 90.0 & 0.77 & 0.63 \\
DeepUbi & 91.83 & 90.12 & 93.55 & 0.9093 & 0.84 \\
hCKSAAP_UbSite & - & - & - & 0.77 & - \\
DeepUbi & 94.10 & 92.31 & 95.89 & 0.9289 & 0.88 \\
iUbiq-Lys & 82.14 & 80.56 & 99.39 & - & 0.50 \\
DeepUbi & 98.92 & 98.90 & 98.93 & 0.9913 & 0.98 \\
ESA-UbiSite & 94.0 & 96.0 & 92.0 & - & 0.92 \\
DeepUbi & 95.59 & 95.53 & 95.65 & 0.9947 & 0.91 \\
\hline
\end{tabular}

\section{Methods}

Benchmark dataset

In this study, the ubiquitination data is collected from the PLMD (v3.0, June. 2017) database [32], which is the biggest online data repository designed for protein lysine modification. The original data contains 121,742 ubiquitination sites from 25,103 proteins. If the data contains homologous samples, it would increase the bias of results. We remove the redundant protein sequences to eliminate homology bias using the CD-HIT web server [33], which is freely available at http://weizhongli-lab.org/cd-hit/, and obtains 12,053 different proteins with $\leq 30 \%$ sequence identity. A sliding window with the length of $15 \times 2+1=31$ is used to intercept the protein sequences with lysine residues in the centre. If the upstream or downstream residues of a protein are less than 15 , the lacking residue is filled with a "pseudo" residue ' $\mathrm{X}$ '. There are too many negative peptides compared to the positive peptides. To obtain a better predictor, we select the negative samples by deleting the redundant segments using $30 \%$ identity to ensure that none of the segments had $\geq 30 \%$ pair-wise identity in the negative peptides [24]. Finally, we obtain a training dataset containing 53,999 ubiquitination and 50,315 non-ubiquitination fragments. A detailed flow chart of these steps is shown in Fig. 4.

\section{Feature construction}

A good feature can extract the correlation of instinct ubiquitination characters and the targets from peptide sequences [34]. Four better feature encoding schemes are adopted, One-Hot encoding, the physicochemical properties, the composition of $\mathrm{k}$-spaced amino acid pairs and the pseudo amino acid composition.

One-Hot Encoding.

The conventional feature representation of amino acid composition uses 20 binary bits to represent an amino acid. To deal with the problem of sliding windows spanning out of the N-terminal or C-terminal, one additional bit is appended to indicate this situation. Then, a vector of size 

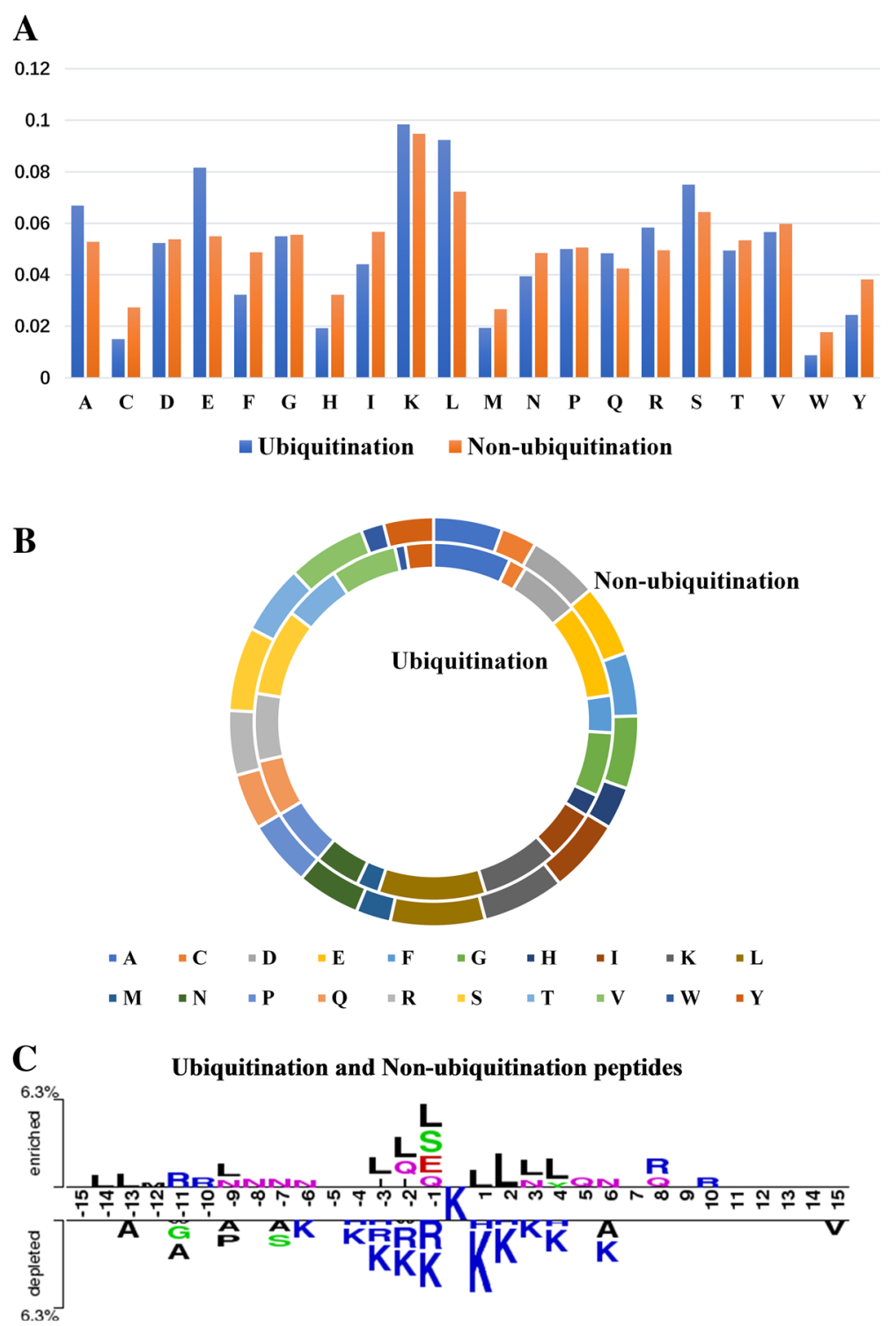

Fig. 3 Different sequence analysis charts about ubiquitination and non-ubiquitination peptides. a A bar chart to compare the number of flanking amino acids surrounding the ubiquitination and non-ubiquitination peptides. $\mathbf{b}$ A circular chart to compare the percentage of flanking amino acids surrounding the ubiquitination and non-ubiquitination peptides. c Two Sample Logos web-server to calculate and visualize differences between ubiquitination and non-ubiquitination peptides

$(20+1)$ bits is used to represent a sample. For example, the amino acid A is represented by ' 100000000000000000000 ' and $\mathrm{R}$ is represented by '010000000000000000000'.

\section{Informative physicochemical properties (IPCP)}

In PTM site prediction, physicochemical properties are essential to extract information for a fragment or protein. Tung [12] proposed an informative physicochemical property mining algorithm that could quantify the effectiveness of individual physicochemical properties in prediction. They used the value of the main effect difference (MED) [35] to estimate the individual effects of physicochemical properties. The property with the largest MED is the most effective in predicting ubiquitination sites. In the study, 31 informative physicochemical properties are selected as the features for calculation, and are listed in Additional file 1: Table S1.

\section{Compositions of $K$-spaced amino acid pairs (CKSAAP)}

The CKSAAP encoding scheme is the composition of $k$-spaced residue pairs (separated by $k$ amino acids) in the protein sequence, which is useful for predicting protein flexible or rigid regions [36]. For example, there are 


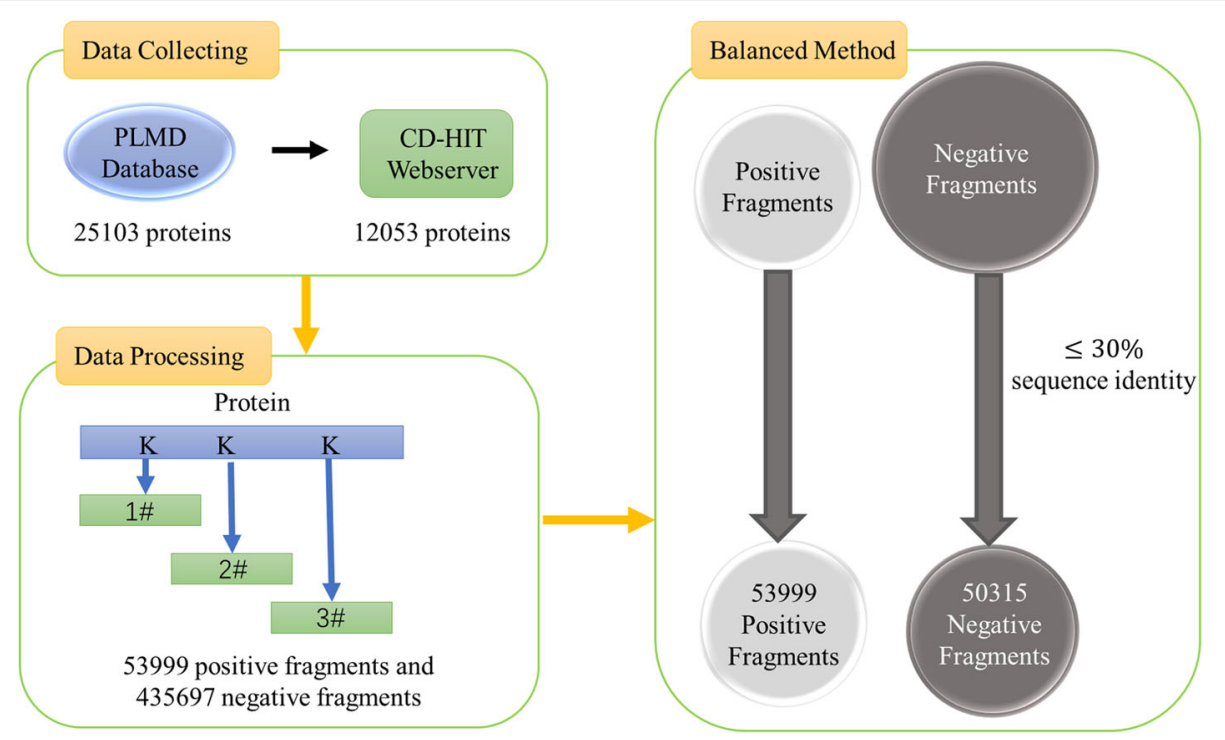

Fig. 4 Flow chart of the data collection and processing. Firstly, collecting the raw proteins and then removing the redundant protein sequences with CD-Hit; secondly, intercepting the protein sequences with a 31 sliding window to get the positive and negative fragments; at last, using $30 \%$ identity in negative samples to get a balanced training data

441 residue pairs (i.e., AA, AC, ..., XX). Therefore, the feature vector can be defined as

$$
\left\{\frac{N_{A A}}{N_{\text {total }}} \quad \frac{N_{A C}}{N_{\text {total }}}, \cdots, \frac{N_{X X}}{N_{\text {total }}}\right\}
$$

where $N_{\text {total }}$ is the total number of $k$-spaced residue pairs in the fragment and $N_{A A}$ is the number of amino acid pair AA in the fragment. Each component in the vector represents the contribution of $k$-spaced amino acid pairs. For instance, the AA component is represented as $\frac{N_{A A}}{N_{\text {total }}}$. In this paper, $k=0,1,2,3,4$, and a $441 \times 5=2205$ vector was obtained by the CKSAAP encoding scheme.

Pseudo Amino Acid Composition (PseAAC).

Chou's pseudo amino acid composition is a set of discrete serial correlation factors combined with traditional 20 amino acid components [37]. In the study, we select 20 correlation factors and the weight of these factors is 0.05 , and a 40 -dimension vector is acquired.

\section{Algorithm}

Deep learning, which evolved from the acquisition of big data, and the power of parallel and distributed computing have facilitated major advances in numerous domains such as image recognition, speech recognition, and natural language processing [38]. Every protein is a sentence, and residues in the protein sequence can be seen as "words". The prediction of ubiquitination can be seen as a 'natural language prediction' (NLP) task. Therefore, we propose a convolutional neural network $(\mathrm{CNN})$ deep learning model and obtain good prediction performance on a large data set. A convolutional neural network $(\mathrm{CNN})$ is a deep learning framework. It is composed of a convolutional layer, a nonlinear layer and a pooling layer. Our model is constructed with six steps (Input a fragment, Construct an embedding layer, Build multi-convolution-pooling layers, Add features, Construct fully connected layers, and an Output layer), as shown in Fig. 5a.

The input protein fragment representation is $x \in R^{L \times}$ ${ }^{21}$, where $L$ is the length of the fragment. The first layer is the embedding layer, which maps input vectors into low-dimensional vector representations. It is essentially a lookup table that we learn from data. $\mathrm{E}=x W_{e}$, where $e$ is the embedding dimension, $W_{e}$ is the embedding weight matrix and $\mathrm{E} \in R^{L \times e}$ is the embedding matrix, which is a continuous product. Then, we assign the embedding matrix $\mathrm{E}$ as an image and use the convolutional neural network to extract features. Because the adjacent residues in the fragments are always highly correlated, one dimensional convolution can be used. The width of the convolution kernel is the dimension of the embedding vector. The height is a super parameter, which is a manual set. For example, if there is a convolution filter with size $a_{k}$, then a feature map is obtained by the convolution

$$
\mathrm{z}_{k}(m)=f\left(\sum_{i=1}^{a_{k}} \sum_{j=1}^{e} w(i, j) \times E(i+m, j)\right)
$$

where $f$ is the activation function, which is a rectified linear unit (ReLU) [39], $w$ is the weight vector and $z_{k} \in$ $R^{L-a_{k}+1}$. The number of convolution filters of size $a_{k}$ is 


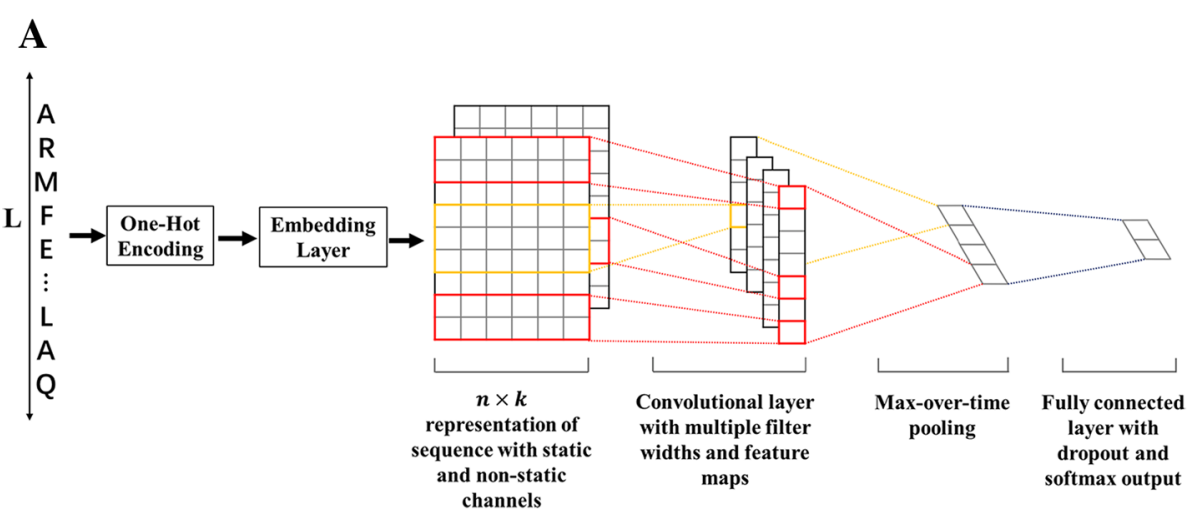

B

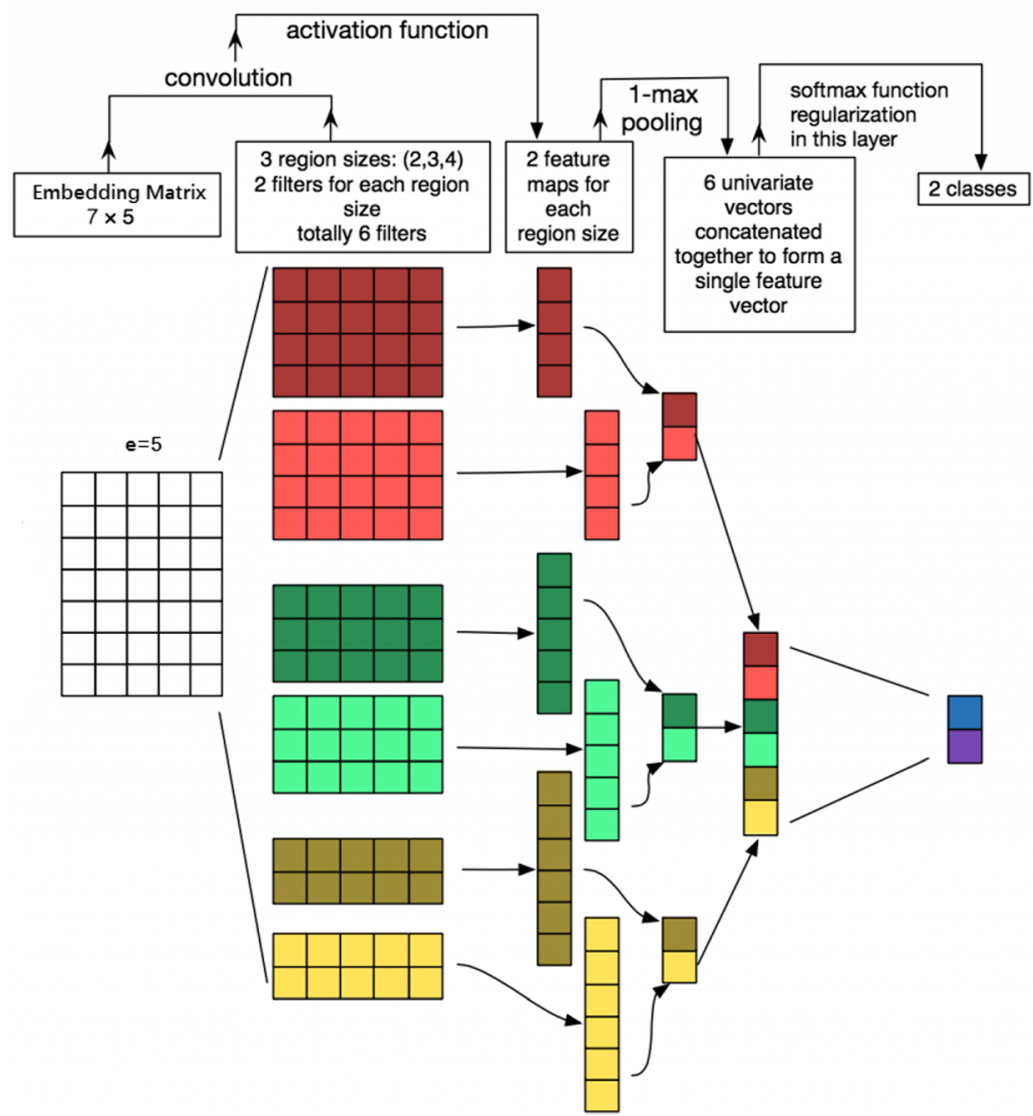

Fig. 5 a Flow chart of the CNN deep learning model. $\mathbf{b}$ An example of convolution-pooling structure. a Input a fragment and encode; construct an embedding layer; build multi-convolution-pooling layers; construct fully connected layers; and then get the output. b Use different filters with different sizes to get a series of feature maps; and then use a max-pooling and concatenating together to form a feature vector. Finally, the softmax function regularization is used to get the classification

also set. The feature map obtained from different convolution kernels is a different size, so a max-pooling function is use to maintain the same dimension. The final eigenvector $h$ is then obtained. For more intuitive understanding, see Fig. 5b. For the first model, CNNUbi, we use the features obtained from the last step without additional features, i.e., $h_{\text {new }}=h$. For comparison, the second model, DeepUbi, is built with additional features and $h_{\text {new }}=[h, b]$, where $b$ is the additional features. Finally, each of the two output units has a score between 0 and 1 , illustrating by the softmax equation $p_{i}=\frac{e^{i}}{\sum_{j} e^{i}}$. Here, $i$ $=F_{c} w_{o}$ represents the input of class unit $i, F_{c}$ is the output of the fully connected layer and $w_{o}$ is the weight 
matrix. The cross-entropy objective function is assigned as the cost function Add features

$$
\begin{aligned}
\mathrm{CE}= & -\sum_{n=1}^{N} y^{n} \ln P\left(y^{n}=1 \mid x^{n}\right) \\
& +\left(1-y^{n}\right) \ln P\left(y^{n}=0 \mid x^{n}\right)
\end{aligned}
$$

where $N$ represents the batch size of the training set and $x^{n}$ and $y^{n}$ represent the $n$-th protein fragment and its label, respectively. Using the Adam optimizers, DeepUbi is trained based on a variety of super-parameters such as the batch size, maximum epoch, learning rate, dropout rate and convolution blocks.

\section{Model evaluation and performance measures}

A confusion matrix is a visual display tool for evaluating the quality of classification models. Each column of the matrix represents the sample situation of the model prediction and each row of the matrix represents the actual situation of the sample. There are four values in the matrix, where TP represents the number of true positives, TN is the number of true negatives, FP is the number of false positives, and FN is the number of false negatives. In the literature, the following metrics based on the confusion matrix are often used to evaluate the performance of a predictor

$$
\left\{\begin{array}{c}
\mathrm{Sp}=\frac{T N}{T N+F P} \\
\mathrm{Sn}=\frac{T P}{F N+T P} \\
\mathrm{Acc}=\frac{T P+T N}{T P+T N+F P+F N} \\
\mathrm{MCC}=\frac{T P \times T N-F P \times F N}{\sqrt{(T P+F N)(T N+F P)(T P+F P)(T N+F N)}}
\end{array}\right.
$$

where $S n$ represents the sensitivity, $S p$ is the specificity, $A c c$ is the accuracy, and $M C C$ is the Matthew's correlation coefficient. The ROC (Receiver Operating Characteristic) curves and the area under the ROC curve (AUC) are usually used to evaluate the classifier's resolving power.

\section{Additional file}

Additional file 1: Table S1. The 31 informative physicochemical properties and their corresponding MED (main effect difference) scores. (XLSX $42 \mathrm{~kb}$ )

\section{Abbreviations}

Acc: Accuracy; AUC: Area under the ROC curve; CKSAAP: Composition of kspaced amino acid pairs; CNN: Convolutional neural network; IPCP: Informative physicochemical properties; MCC: Mathew's correlation coefficient; MED: Main effect difference; PseAAC: Pseudo amino acid composition; PTM: Post-translational modification; RBF: Radial basis function: ReLU: Rectified linear unit; Sn: Sensitivity; Sp: Specificity; SVM: Support vector machine

\section{Acknowledgements}

Dr. Jun Ding helped us with the programming and processed the data. We also thanked the anonymous reviewers who gave us very valuable suggestions. The manuscript is edited by American Journal Experts (AJE) prior to submission.

\section{Funding}

This work is supported by grants from the Natural Science Foundation of China (11671032) and the 2015 National Traditional Medicine Clinical Research Base Business Construction Special Topics (JDZX2015299). The funders have no role in the design of the study, collection, analysis, and interpretation of the data or writing the manuscript.

\section{Availability of data and materials}

A total of 121,742 ubiquitination sites were collected from PLMD database (http://plmd.biocuckoo.org/) and the proteins were retrieved from UniProt (https://www.uniprot.org/). The data is provided on website https:// github.com/Sunmile/DeepUbi and the file name is "Raw Data".

\section{Authors' contributions}

$Y X$ and $Y Y$ conceived of and designed the experiments. HF, XW, HW and YY performed the experiments and data analysis. HF and $Y X$ wrote the paper. $Y X$ and $Y Y$ revised the manuscript. All authors read and approved the final manuscript.

Ethics approval and consent to participate

Not applicable.

Consent for publication

Not applicable.

Competing interests

The authors declare that they have no competing interests.

\section{Publisher's Note}

Springer Nature remains neutral with regard to jurisdictional claims in published maps and institutional affiliations.

\section{Author details}

${ }^{1}$ Department of Information and Computing Science, University of Science and Technology Beijing, Beijing 100083, China. ${ }^{2}$ Institute of Computing Technology, Chinese Academy of Sciences, Beijing 100190, China. ${ }^{3}$ Beijing Key Laboratory for Magneto-photoelectrical Composite and Interface Science, University of Science and Technology Beijing, Beijing 100083, China.

Received: 8 November 2018 Accepted: 12 February 2019

Published online: 18 February 2019

\section{References}

1. Goldstein G, Scheid M, Hammerling U, Schlesinger DH, Niall HD, Boyse EA. Isolation of a polypeptide that has lymphocyte-differentiating properties and is probably represented universally in living cells. Proc Natl Acad Sci U S A. 1975;72(1):11-5.

2. Wilkinson KD. Protein ubiquitination: a regulatory post-translational modification. Anticancer Drug Des. 1987;2(2):211-29.

3. Ou CY, Pi HW, Chien CT. Control of protein degradation by E3 ubiquitin ligases in Drosophila eye development. Trends Genet. 2003;19(7):382-9.

4. Herrmann J, Lerman LO, Lerman A. Ubiquitin and ubiquitin-like proteins in protein regulation. Circ Res. 2007;100(9):1276-91.

5. Welchman R, Gordon C, Mayer RJ. Ubiquitin and ubiquitin-like proteins as multifunctional signals. Nat Rev Mol Cell Biol. 2005;6(8):599-609.

6. Hurley JH, Sangho L, Gali P. Ubiquitin-binding domains. Biochem J. 2006; 399(Pt 3):361.

7. Nath D, Shadan S. The ubiquitin system. Nature. 2009;458(7237):421-21.

8. Schwartz AL, Ciechanover A. The ubiquitin-proteasome pathway and pathogenesis of human diseases. Annu Rev Med. 1999;50:57-74.

9. Zhong J, Shaik S, Wan L, Tron AE, Wang Z, Sun L, Inuzuka H, Wei W. SCF beta-TRCP targets MTSS1 for ubiquitination-mediated destruction to regulate cancer cell proliferation and migration. Oncotarget. 2013;4(12): 2339-53.

10. Hitchcock AL, Kathryn A, Gygi SP, Silver PA. A subset of membraneassociated proteins is ubiquitinated in response to mutations in the endoplasmic reticulum degradation machinery. Proc Natl Acad Sci U S A. 2003;100(22):12735-40.

11. Ikeda F, Dikic I. Atypical ubiquitin chains: new molecular signals. EMBO Rep. 2008;9(6):536-42. 
12. Tung CW, Ho SY: Computational identification of ubiquitylation sites from protein sequences. Bmc Bioinformatics 2008, 9(1):310-310.

13. Kawashima S, Pokarowski P, Pokarowska M, Kolinski A, Katayama T, Kanehisa M. AAindex: amino acid index database, progress report 2008. Nucleic Acids Res. 2008;36(Database issue):D202-5.

14. Radivojac P, Vacic V, Haynes C, Cocklin RR, Mohan A, Heyen JW, Goebl MG, lakoucheva LM. Identification, analysis, and prediction of protein ubiquitination sites. Proteins. 2010;78(2):365-80.

15. Zhao X, Li X, Ma Z, Yin M. Prediction of lysine ubiquitylation with ensemble classifier and feature selection. Int J Mol Sci. 2011;12(12):8347-61.

16. Lee TY, Chen SA, Hung HY, Ou YY. Incorporating distant sequence features and radial basis function networks to identify ubiquitin conjugation sites. PLoS One. 2011;6(3):e17331.

17. Chen Z, Chen YZ, Wang XF, Wang C, Yan RX, Zhang ZD. Prediction of ubiquitination sites by using the composition of $\mathrm{k}$-spaced amino acid pairs. PLoS One. 2011;6(7).

18. Cai YD, Huang T, Hu LL, Shi XH, Xie L, Li YX. Prediction of lysine ubiquitination with mRMR feature selection and analysis. Amino Acids. 2012; 42(4):1387-95.

19. Chen X, Qiu JD, Shi SP, Suo SB, Huang SY, Liang RP. Incorporating key position and amino acid residue features to identify general and speciesspecific ubiquitin conjugation sites. Bioinformatics. 2013;29(13):1614-22.

20. Chen Z, Zhou Y, Song JN, Zhang ZD. hCKSAAP UbSite: improved prediction of human ubiquitination sites by exploiting amino acid pattern and properties. Bba-Proteins Proteom. 2013;1834(8):1461-7.

21. Qiu WR, Xiao X, Lin WZ, Chou KC. iUbiq-Lys: prediction of lysine ubiquitination sites in proteins by extracting sequence evolution information via a gray system model. J Biomol Struct Dyn. 2015;33(8): 1731-42.

22. Cai $B$, Jiang X. Computational methods for ubiquitination site prediction using physicochemical properties of protein sequences. BMC Bioinformatics. 2016;17:116

23. Wang JR, Huang WL, Tsai MJ, Hsu KT, Huang HL, Ho SY. ESA-UbiSite: accurate prediction of human ubiquitination sites by identifying a set of effective negatives. Bioinformatics. 2017;33(5):661-8.

24. Huang C-H, Su M-G, Kao H-J, Jhong J-H, Weng S-L, Lee T-Y. UbiSite: incorporating two-layered machine learning method with substrate motifs to predict ubiquitin-conjugation site on lysines. BMC Syst Biol. 2016;10(1):S6.

25. Li Y, Xie P, Lu L, Wang J, Diao L, Liu Z, Guo F, He Y, Liu Y, Huang Q, et al. An integrated bioinformatics platform for investigating the human E3 ubiquitin ligase-substrate interaction network. Nat Commun. 2017;8(1):347.

26. Walsh I, Di Domenico T, Tosatto SCE. RUBI: rapid proteomic-scale prediction of lysine ubiquitination and factors influencing predictor performance. Amino Acids. 2014;46(4):853-62.

27. Kai-Yan F, Tao H, Kai-Rui F, Xiao-Jun L. Using WPNNA classifier in ubiquitination site prediction based on hybrid features. Protein Pept Lett. 2013:20(3):318-23.

28. Nguyen V, Huang $K$, Huang $C$, Lai KR, Lee T. A new scheme to characterize and identify protein ubiquitination sites. IEEE/ACM Trans Comput Biol Bioinform. 2017;14(2):393-403.

29. Ghosh S, Febin Prabhu Dass J. Non-canonical pathway network modelling and ubiquitination site prediction through homology modelling of NF-KB. Gene. 2016:581(1):48-56.

30. Chou KC. Some remarks on protein attribute prediction and pseudo amino acid composition. J Theor Biol. 2011:273(1):236-47.

31. Vacic V, lakoucheva LM, Radivojac P. Two sample logo: a graphical representation of the differences between two sets of sequence alignments. Bioinformatics. 2006;22(12):1536-7.

32. Xu H, Zhou J, Lin S, Deng W, Zhang Y, Xue Y. PLMD: an updated data resource of protein lysine modifications. J Genet Genomics. 2017;44(5):243-50.

33. Huang Y, Niu BF, Gao Y, Fu LM, Li WZ. CD-HIT suite: a web server for clustering and comparing biological sequences. Bioinformatics. 2010;26(5): 680-2.

34. Plewczynski D, Tkacz A, Wyrwicz LS, Rychlewski L. AutoMotif server: prediction of single residue post-translational modifications in proteins. Bioinformatics. 2005;21(10):2525-7.

35. Tung CW, Ho SY. POPI: predicting immunogenicity of MHC class I binding peptides by mining informative physicochemical properties. Bioinformatics. 2007;23(8):942-9.

36. Chen $K$, Kurgan LA, Ruan J. Prediction of flexible/rigid regions from protein sequences using k-spaced amino acid pairs. BMC Struct Biol. 2007;7:25.
37. Chou KC. Prediction of protein cellular attributes using pseudo-amino acid composition. Proteins-Structure Function and Genetics. 2001:43(3):246-55.

38. Min S, Lee B, Yoon S. Deep learning in bioinformatics. Brief Bioinform. 2017; 18(5):851-69.

39. Nair $\mathrm{V}$, Hinton GE. Rectified linear units improve restricted boltzmann machines. In: International conference on international conference on machine learning; 2010. p. 807-14.

\section{Ready to submit your research? Choose BMC and benefit from:}

- fast, convenient online submission

- thorough peer review by experienced researchers in your field

- rapid publication on acceptance

- support for research data, including large and complex data types

- gold Open Access which fosters wider collaboration and increased citations

- maximum visibility for your research: over $100 \mathrm{M}$ website views per year

At $\mathrm{BMC}$, research is always in progress.

Learn more biomedcentral.com/submissions 